\title{
REVIEW
}

\section{Neuroanatomy and neurophysiology related to sexual dysfunction in male neurogenic patients with lesions to the spinal cord or peripheral nerves}

\author{
K Everaert ${ }^{1}$, WIQ de Waard ${ }^{1}$, T Van Hoof ${ }^{2}$, C Kiekens $^{3}$, T Mulliez ${ }^{1}$ and C D'herde ${ }^{2}$ \\ ${ }^{1}$ Department of Urology, Ghent University Hospital, Ghent, Belgium; ${ }^{2}$ Department of Anatomy, Embryology, Histology \\ and Medical Physics, University of Ghent, Ghent, Belgium and ${ }^{3}$ Department of Rehabilitation Sciences, Catholic University Leuven, \\ Leuven, Belgium
}

\begin{abstract}
Study design: Review article.
Objectives: The neuroanatomy and physiology of psychogenic erection, cholinergic versus adrenergic innervation of emission and the predictability of outcome of vibration and electroejaculation require a review and synthesis.

Setting: University Hospital Belgium.

Methods: We reviewed the literature with PubMed 1973-2008.

Results: Erection, emission and ejaculation are separate phenomena and have different innervations. It is important to realize, which are the afferents and efferents and where the motor neuron of the end organ is located. When interpreting a specific lesion it is important to understand if postsynaptic fibres are intact or not. Afferents of erection, emission and ejaculation are the pudendal nerve and descending pathways from the brain. Erection is cholinergic and NO-mediated. Emission starts cholinergically (as a secretion) and ends sympathetically (as a contraction). Ejaculation is mainly adrenergic and somatic. For vibratory-evoked ejaculation, the reflex arch must be complete; for electroejaculation, the postsynaptic neurons (paravertebral ganglia) must be intact.

Conclusion: Afferents of erection, emission and ejaculation are the pudendal nerve and descending pathways from the brain. Erection is cholinergic and NO-mediated. Emission starts cholinergically (as a secretion) and ends sympathetically (as a contraction). Ejaculation is mainly adrenergic and somatic. In neurogenic disease, a good knowledge of neuroanatomy and physiology makes understanding of sexual dysfunction possible and predictable. The minimal requirement for the success of penile vibration is a preserved reflex arch and the minimal requirement for the success of electroejaculation is the existence of intact post-ganglionic fibres.
\end{abstract}

Spinal Cord (2010) 48, 182-191; doi:10.1038/sc.2009.172; published online 5 January 2010

Keywords: erection; ejaculation; neurogenic patients; neuroanatomy; neurophysiology

\section{Introduction}

Sexual dysfunction is a complication of neurogenic diseases (such as spina bifida, spinal cord injury, or multiple sclerosis, among others) and erection is less prone to dysfunction as compared with ejaculation. ${ }^{1-4}$ Higher spinal lesions maintain penile responses to reflexogenic stimulation, whereas lower lesions maintain psychogenic-mediated reflex erection. In addition, emission is maintained in most patients, even in lower and peripheral lesions. Ejaculation is more often impaired and preserved in $4 \%$ of the higher and $18 \%$ of the lower lesions, but can be evoked in roughly

Correspondence: Dr K Everaert, Department of Urology, Universital Ghent Belgium, De Pintelaan 185, Ghent, Oost Vlaanderen 9000, Belgium. E-mail: K.Everaert@ugent.be

Received 26 May 2009; revised 2 November 2009; accepted 15 November 2009; published online 5 January 2010
$80 \%$ of the patients with penile vibration and in $90 \%$ with electroejaculation. ${ }^{4-6}$ Orgasm is preserved in $40-50 \%$ of the patients. $^{7}$

Autonomic innervations are summarized as a sympathetic thoracolumbar pathway responsible for ejaculation and a lower sacral pathway for erection. In the literature, complete spinal lesions are classified as the pathways mentioned above, damage of the thoracolumbar or sacral level, damage in between both pathways and peripheral lesions. ${ }^{4}$ Clinical patterns, questionnaires or laboratory findings have been related to these anatomical double pathways, but lack detailed implementation of the knowledge of anatomy. Psychogenic erection, cholinergic versus adrenergic innervation of emission and the predictability of outcome of vibration and electroejaculation are issues, which require anatomical review and synthesis. 
The aim of this review is to describe in a comprehensible and detailed manner how neuroanatomy is related to different nervous tissue lesions, how different lesions affect their end organs and relate this to therapy. Misconceptions on sympathetic psychogenic erections, dual innervations of ejaculation, sexual dysfunction in spinal lesions, orgasm in spinal lesions and orgasm after cystoprostatectomy are discussed.

\section{Materials and methods}

We reviewed the literature (PubMed 1973-2008) on the neuroanatomy and neurophysiology of erection and ejaculation and on therapy for neurogenic patients and their dysfunctions. Search terms were spinal cord, erection, ejaculation, emission and neurogenic.

\section{Results}

\section{Erection}

Introduction. Erection is the result of a high parasympathetic and low sympathetic tone resulting in the relaxation of the smooth muscle cells of the corpora cavernosa and the helicine penile arteries. The result is an increased arterial inflow and an increased resistance to outflow due to compression on the veins against the tunica albuginea; consequently the intracavernous pressure increases (80$100 \mathrm{mg} \mathrm{Hg}$ ). Once rigidity is obtained, the inflow decreases significantly and rigidity is conserved with low flow (3-5 mg/ min) until the parasympathetic inflow decreases or the orthosympathetic tone increases. ${ }^{8}$

Neuroanatomy and physiology of erection. Afferents: The pudendal nerve conducts sensory information from the genital, perineal, perianal and scrotal skin to the sacral cord (S2-S3-S4) (Figure 1). The scrotum also has higher (lumbar) sensory innervations: the ilioinguinal nerve L1 and the genital branch of the genitofemoral nerve L1-L2. Notwithstanding this, anatomical dissection studies show the heterogeneity of cutaneous branches of these nerves. The classical pattern of distribution and the terminal course of the ilioinguinal and genitofemoral nerve consistent with modern text descriptions were defined in only $20 \%$ of cadaveric dissections. ${ }^{9,10}$ The dorsal penile nerve (pudendal nerve) is responsible for reflex erections. ${ }^{11}$ Damage to the pudendal nerves (that is, Alcock syndrome) leads to erectile dysfunction. ${ }^{12}$ In complete spinal cord injury above the sacral centres there are no means to evaluate sensory innervation by means of pinprick, temperature or cotton swab testing because of lack of transmission of the signals through the spinal cord. The bulbocavernous reflex remains intact in these patients and is the clinical test to perform to evaluate the pudendal nerve integrity.

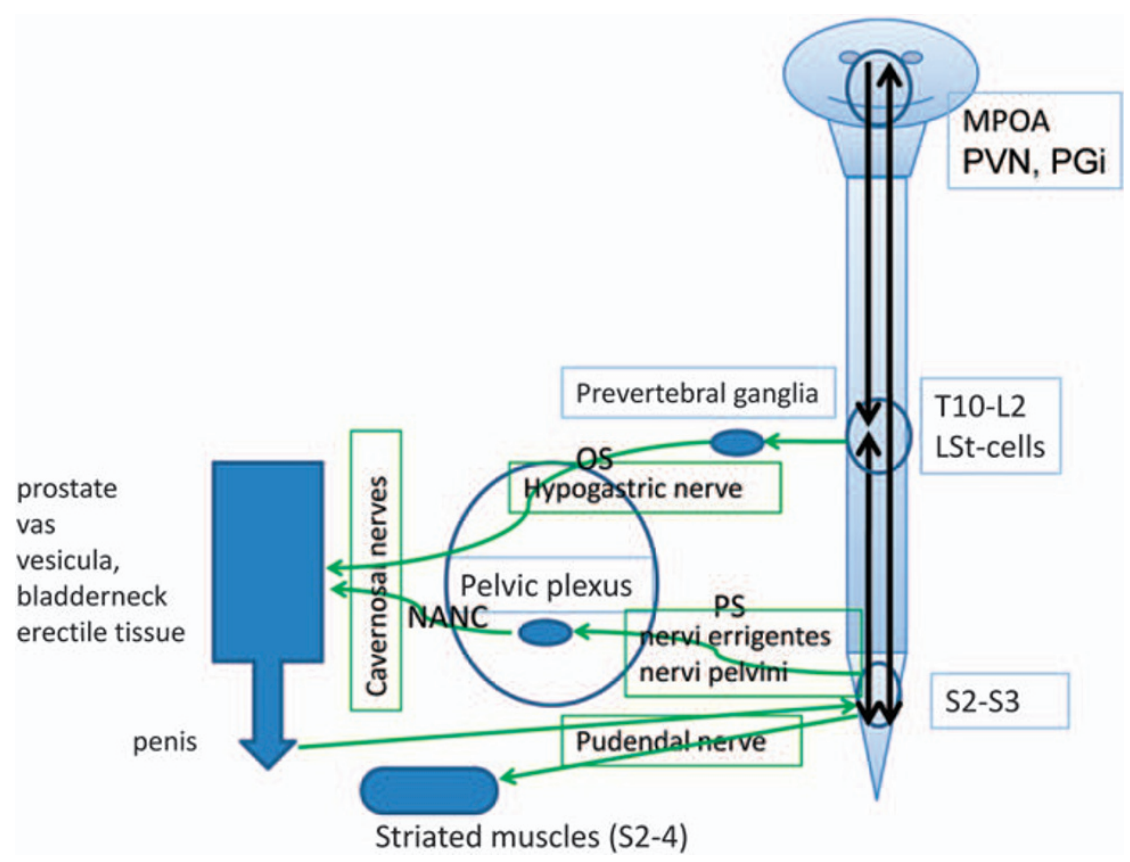

Figure 1 Neuroanatomy of reflex and psychogenic erection, emission and ejaculation in a normal individual. (1) Pudendal stimulation causes reflex parasympathetic erection through activation of the parasympathetic pathway (pelvic nerves and NANC fibres). (2) Auditory, visual and olfactory pathways, fantasy and dreams can induce psychogenic parasympathetic erection (pelvic and NANC nerves). (3) Anxiety, stress, cerebral inhibition can depress erection through activation of the sympathetic pathway (hypogastric nerves). (4) Contraction =flacidity $=$ adrenergic stimulation, Hypogastric nerve (OS). Relaxation =erection =NO stimulation (NANC), Acetylcholine facilitates (PS). Erection depends on equilibrium between the OS and NANC stimulation. (5) Emission starts cholinergic (secretion) and ends sympathetic (contraction bladder neck, vas, seminal vesicles, prostate, ... ). (6) Ejaculation is mainly adrenergic and somatic. The spinal reflex ejaculation generator centre has been localized in the thoracolumbar region and contains lumbar spinothalamic cells (LSt-cells). (7) Some sacral innervated muscles like the levator muscles, the urethral and anal external sphincters are innervated both by the pudendal and pelvine nerves. 
In addition, central afferent stimulation (tactile (from higher dermatomes), audiovisual or olfactory and psychogenic (fantasy, emotion) stimuli) can provoke erection as is seen in psychogenic erection. ${ }^{13}$ The psychogenic erection is also suggested to be responsible for sustained erection during intercourse, explaining why some spinal cord-injured patients obtain good reflex erections but have problems sustaining erection.

Efferents: Nuclei of parasympathetic neurons are localized in the sacral spinal cord(S2-S4). The efferent impulses are conducted to the corpora cavernosa through preganglionar (long) pelvic splanchnic nerves or 'pelvic nerves' or nervi erigentes, and through short post-ganglionar nervi cavernosi, also called non-adrenergic non-cholinergic (NANC) fibres ${ }^{13-15}$ (Figure 1). These NANC fibres have nitric oxide (NO) as a neurotransmitter, whereas the long cholinergic fibres have acetylcholine as a neurotransmitter. NANC fibres have the most important role in the erection, whereas acetylcholine is found to have a modulating role by facilitating the release of NO from the NANC fibres and inhibiting noradrenaline release from the sympathetic fibres. Furthermore, acetylcholine contracts penile smooth muscle in vitro, and erection is resistant to the cholinergic antagonist atropine. Acetylcholine also facilitates NO release by activating endothelial cells. NANC fibres also release VIP (vasoactive intestinal polypeptide), whose relaxant effect has been shown. ${ }^{15}$ In addition to the peripheral endings, autonomic sensory fibres are capable of releasing neuropeptides (for example, substance $P$ and calcitonin gene-related peptide), which display vasorelaxant effects in vitro. But their physiological role in the control of erection remains to be demonstrated. ${ }^{14,16}$

The nuclei of the orthosympathetic neurons are localized in the columnae intermediolateralis of the thoracolumbar spinal cord (T11-12). Their short preganglionar fibres run through the prevertebral sympathetic chain and ganglions; there the long post-ganglionic fibres run through the hypogastric nerve, the pelvic plexus and the cavernous nerves to the corpora cavernosi where they inhibit erection. ${ }^{17,18}$ The sympathetic post-ganglionic axons may reach the penis by two additional routes. The first is the paravertebral sympathetic chain, which connects via the pelvic nerve to the pelvic plexus and then via the cavernous nerve to the penis. The second pathway is the paravertebral sympathetic chain via the pudendal nerve to the penis. ${ }^{14}$

The central and peripheral originating stimuli are processed cortically and conducted through the hypothalamus to the thoracolumbar and sacral spinal cord centres, where they respectively decrease hypothetically the orthosympathetic and increase the parasympathetic tone. In rats, as well, the bilateral section of the hypogastric or the pelvic nerves does not result in complete erectile dysfunction. ${ }^{19}$ Finally, there was no decrease in the mounting behaviour of rats after the complete transection of both hypogastric nerves and a brief noncontact exposure of males to receptive females. ${ }^{19,20}$ On the other hand, patients with complete lesions of the sacral cord report psychogenic erections, whereas when parasympathetic motor neurons are damaged there should be no more erection. ${ }^{13,21}$ Following pre-ganglionic nerve (autonomic) section, extensive collateral sprout- ing from residual pre-ganglionic fibres or fibres from higher or lower ganglia is seen within 4-5 weeks. ${ }^{22}$ This existence of such an extensive neuroplasticity in the prevertebral ganglia means that even in complete destruction of the sacral spinal cord a certain 'NANC tone' can exist, and with it a certain relaxation (not erection) of the cavernous smooth muscles is preserved; when the sympathetic fibres cease firing, a decrease in contraction of the cavernous muscles can occur. It has been suggested that this supraspinal vasodilator pathway is a sympathetic cholinergic pathway, operating through cholinergic (NANC) neurons in the pelvic plexus. ${ }^{23}$ Sympathetic fibres are found to take synapses in the pelvic plexus with NANC fibres, suggesting another mechanism for how patients with sacral lesions may have hypothetically psychogenic erections. Notwithstanding the fact that the sympathetic nervous system is considered to be the antierectile centre, this pathway may also facilitate erections after an injury to parasympathetic pathways. Dail et al. ${ }^{24}$ stimulated the hypogastric nerve of intact anaesthetised rats and observed no increase in cavernous pressure. However, within 3 days after the unilateral section of the pelvic nerve, stimulation of the ipsilateral hypogastric nerve caused a significant increase in the cavernous pressure. This suggests a plasticity of the nervous system and the experiment shows that in intact rats with normal pelvic nerve function, the hypogastric nerve has little or no role in promoting erection. $^{21,24}$ The observations of Weihe et al. ${ }^{25}$ suggest a mode of autonomic regulation, based on the co-release of norepinephrine and acetylcholine at parasympathocardiac, sudomotor and selected vasomotor neuroeffector junctions, that is unique to the primate's peripheral nervous system.

As mentioned before, the destruction of sacral autonomic centres abolishes reflex erections but not psychogenic erections. The residual erectile pathway may then be mediated by thoracolumbar sympathetic outflow. In patients with lesions above T9, psychogenic erections are eliminated but reflex erections persist. However, it remains unclear whether thoracolumbar sympathetic outflow participates in psychogenic erections in neurally intact men. ${ }^{13,14,16}$ This orthosympathetic-originated erection has been studied in rats: there is maintenance of erection of the penile glans, but not the penile body, after the transection of rats' cavernous nerves.

Central regulation of the erection: The central regulation of erection is not within the scope of this article, but is summarized below as it has its clinical use. ${ }^{13}$

Catecholamines in the central nervous tissue increase sexual activity and erection (L-dopa, amphetamines, monoamine oxidase inhibitors) through $\alpha-2$ receptors (clonidine causes inhibition, yohimbine leads to stimulation). This is clinically relevant in the treatment of patients with Parkinson's disease.

Dopamine stimulates sexual function by D1 and D2 receptors. This is clinically relevant in the treatment of patients with Parkinson's disease. Stimulation of the dopaminergic neurons of the medial proptic area leads to the activation of oxcytocinergic neurons (hypothalamus) and consequently to erection. Indeed, erection is inhibited as well by antagonists of dopamine such as oxytocin. Prolactin 
inhibits sexual function indirectly through the dopaminergic system. Stimulation of the dopaminergic system (apomorfine) has been used with moderate effect in the treatment of erectile dysfunction.

Serotonin is generally the inhibiting neurotransmitter of sexual function in the brain and the spinal cord and, as such, of the erection. This is best illustrated through the side effects of SSRIs in depression therapy. However, the effect depends on the serotonin subtype receptor. Melatonin stimulates sexual function indirectly through the serotoninergic system.

The erectile reflex: Afferents are the pudendal nerve or the brain. The contraction of the cavernous body is adrenergic stimulation and leads to flaccidity (hypogastric nerve (OS)). Relaxation of the cavernous body is NO stimulation and leads to erection (NANC); acetylcholine facilitates (PS). Erection depends on an equilibrium between the OS and NANC stimulation.

Strong parasympathetic stimulation (reflex erection and psychogenic erection) and low orthosympathetic tone (psychogenic erection) cause erection and start emission (see later because of increased parasympathetic secretion). With increasing orthosympathetic stimulation emission is completed (orthosympathetic contraction, see later) and followed by ejaculation (orthosympathetic contraction, see later). The parasympathetic tone decreases in the presence of a maximal orthosympathetic stimulation leading to detumescence of the penis.

\section{Ejaculation}

Introduction. Orgasm is seen in $40-50 \%$ of the spinal lesions. Its appearance is not related to the level of the lesion and is the result of cerebral processing of peripheral and central impulses. ${ }^{26}$

Besides a decreased latency for orgasm ${ }^{7}$ little is known about orgasms in male patients with spinal lesions, the discussion on the neuroanatomy is out of the scope of this publication.

Ejaculation is the expulsion of semen from the penis. During ejaculation, pressures of more than 5 metres water pressure have been found in the prostatic urethra. ${ }^{27}$ When rhythmic contractions of the pelvic muscles (for the purpose of this article defined as 'somatic ejaculation') occur, there will be moments of relaxation of the urethral sphincter, and then semen will leak into the bulbar urethra, dilating the bulbous and resulting in a urethrobulbocavernous or urethro-genital reflex contraction and expelling semen out of the penis. ${ }^{28}$ More recently a 'spinal pattern generator' model was developed and extensively tested to literature findings. ${ }^{29}$ Spinal pattern generators are known to be responsible for several protective reflexes like swallowing and coughing and match perfectly with the rhythmic contraction during ejaculation. ${ }^{30}$ In conclusion, ejaculation has two components, a somatic and an autonomic, which are for the purpose of this article defined as 'somatic ejaculation' and 'autonomic ejaculation'. ${ }^{29}$ Nocturnal ejaculation stands for what could also be called 'psychogenic ejaculation'. At last we are recognizing vibratory ejaculation and electroejaculation both with their specific indications. ${ }^{31,32}$

The ejaculation time varies among men depending on arousal, inflammation, seminal magnesium, penile sensitivity, psychological factors, hormones (testosterone, oxytocine) and the intensity of perineal stimuli. The intraindividual variation of the ejaculation time is constant for masturbation, sexual intercourse and vibratory stimulation in a standardized setting. ${ }^{33}$ Patients with neurogenic diseases mostly suffer from anejaculation or retrograde ejaculation, more rarely from delayed ejaculation. Patients with hyperesthesia in the genital region and patients with incomplete lesions of the conus region of the spinal cord often suffer from premature ejaculation. ${ }^{34}$

Neuroanatomy and physiology of emission and ejaculation. Intrinsic contractile activity of the vas deferens: A rhythmic contractility of the vas deferens is seen during perfusion experiments, and contractions are triggered by increasing pressure (meaning also by increased secretion) rather than by mechanoreceptors (neurons) or afferents (pudendal nerve, hypogastric nerve). Contractility is not altered by the sectioning of nerves, the $\alpha$-receptor blockade, atropine or ganglion blockade, but it is demolished following the perfusion of potassium cyanide. ${ }^{35}$

Afferents: Electrostimulation and vibration of the pudendal nerve provokes ejaculation and can be abolished with a ring anaesthesia of the penis, causing the bulbocavernous reflex to disappear (Figure 1). ${ }^{6,36-38}$ The region of vibratory stimulation in neurogenic patients must not necessarily be the penis; stimulation of the perineum, suprapubic region and scrotum has also been found successful. However, penile vibration is generally accepted as the most effective and the best studied. ${ }^{37,38}$ The presence of a bulbocavernous reflex is predictive for the outcome of vibratory ejaculation. ${ }^{39}$

Experiments with the pseudorabies virus suggest that there are no afferents running with the pelvic or hypogastric nerves. Afferents in the cavernous nerves terminate in Lissauer's tract, the lateral and medial collateral pathways and just dorsal to the parasympathetic pre-ganglionic nucleus (SPN). ${ }^{13}$ It may also be that, during normal erection, there is, in addition to pelvic dilatation, a local decrease of the resting vasoconstrictor tone mediated through a reflex having its afferent pathway in the hypogastric nerve, and the activation of this reflex can produce some tumescence even in the absence of concomitant dilator activation. ${ }^{40}$ Alternatively, sympathetic afferents in hypogastric nerves may merely trigger a reduction in vasoconstrictive sympathetic outflow allowing partial tumescence. ${ }^{13,24,41,42}$

Afferents from the brain must exist and could be responsible, for example, for nocturnal ejaculation (psychogenic ejaculation), but they are not well described in the literature.

Efferents: The motor neurons of the hypogastric nerves are found in the ganglions of the prevertebral sympathetic chain (Figure 1). ${ }^{43}$ Electrostimulation of the hypogastric nerves results in immediate emission and ejaculation. The effect increases with $\alpha$-receptor mimetic drugs and does not alter with $\beta$-receptor-blocking agents. A biphasic contractile 
response (tonic $=$ purinergic; phasic $=$ adrenergic) following electrostimulation has not been confirmed by all authors. Others notice that following electrostimulation, the proximal vas contracts followed 5-10 s later by the distal vas deferens. ${ }^{44}$ Transection of the hypogastric nerve completely abolishes ejaculation following electrostimulation of the penis. ${ }^{43}$ Electrostimulation of the hypogastric nerve also causes contraction of the bladder neck, the prostate, the seminal vesicles and the vas deferens. ${ }^{4-47}$

The hypothesis of cholinergic stimulation (during erection) is based on the observation of 'acetylcholinesterase colouring fibres' in the vas deferens, seminal vesicles and the bladder neck. ${ }^{35}$ Also in spinally transected rats, the administration of muscarine evokes erection and ejaculation and facilitates penile reflexes without any proof that ejaculation is directly facilitated as such. Electrostimulation of the pelvic nerves leads to increased contractility of the vas deferens, and this can be partially blocked with atropine. However, transection of the pelvic nerves does not block ejaculation elicited by electrostimulation of the dorsal penile nerve. Acetylcholine has very little effect on the contractility of the vas deferens, the bladder neck and the prostate. Acetylcholine significantly increases the contractility of the seminal vesicles, and cholinergic stimulation enhances the secretory function of epithelial cells in the male accessory glands (distal epididymis, seminal vesicles, prostate). ${ }^{4-49}$ Seminal vesicles receive dual sympathetic and parasympathetic innervations. Stimulation of the lesser splanchnic nerves induces the contraction of the seminal vesicle in rats. Sympathetic innervation of the seminal vesicle is associated with nonvascular smooth muscle, whereas parasympathetic innervation is associated with the glandular epithelium. ${ }^{41}$ Experiments on the bladder neck and prostate show that the contraction of smooth muscle is adrenergic and not cholinergic. The above-described increase in contractility is therefore suggested to be secondary to an increase in secretion.

Adrenergic stimulation (ephedrine) is useful clinically in neurogenic patients with retrograde ejaculation. ${ }^{1}$ It is obvious in patients with an open bladder neck, but also helpful if they have a closed bladder neck because an increased bladder neck tone will facilitate antegrade ejaculation. ${ }^{50}$

In conclusion: increased cholinergic stimulation (erection) increases the secretion of the distal epididymes, the seminal vesicles and the prostate leading to emission. The emission gains further strength through an increase in adrenergic stimulation with contraction of the vas deferens, the seminal vesicles, smooth muscles from the prostate and the bladder neck. Finally, when high pressures are seen in the prostatic urethra, ejaculation occurs with again important adrenergic activity and rhythmic somatic stimulation of sacral muscles.

Central regulation of ejaculation: The central regulation of the sexual function is not the focus of this review, but for the sake of completeness we summarized the literature. Serotonin (medial preoptic area) mainly causes inhibition of ejaculation through inhibition of the stimulatory dopamine (paraventricular nucleus of the hypothalamus). Cathecholamines (paragigantocellular nucleus) cause stimulation of ejaculation. Brain damage can separately affect emission, ejaculation and orgasm. ${ }^{41}$

The spinal reflex ejaculation generator centre has been localized in the thoracolumbar region and contains lumbar spinothalamic cells (LST cells). ${ }^{29,30}$ These lumbar spinothalamic cells activate (increased c-Fos activity) during ejaculation and are direct projections of cells in the paragigantocellular nucleus in the thalamus. A complete lesion of these lumbar spinothalamic cells leads to anejaculation in rats but does not decrease or abolish mounting or intromission. Animal studies have clearly shown that connections with higher centres are needed to have normal ejaculatory function, but ejaculation is possible without these higher connections.

The ejaculatory reflex: Ejaculation is a spinal reflex elicited by electrostimulation of the dorsal penile nerve (Figure 1). This is best illustrated by the ability of vibratory stimulation of the penis to induce ejaculation in patients with complete spinal cord transection above the tenth thoracic segmental level. The ability of a peripheral stimulation to induce ejaculation despite the complete loss of reciprocal connections with supraspinal structures implies the existence of an ejaculation control centre at the spinal cord level. ${ }^{41}$ In patients with a complete spinal cord lesion, ejaculation is not abolished: $10 \%$ can obtain ejaculation by masturbation and $80 \%$ with vibratory stimulation. ${ }^{5}$ Anaesthesia of the urethra does not destroy the ejaculatory reflex, but anaesthesia of the penile dorsal nerve does. ${ }^{36}$ Evidence exists that increasing intraurethral pressure (emission) in the prostatic urethra leads to ejaculation, but even when emission is blocked (guanethidine) ejaculation remains unchanged with sexual activity or electrostimulation of the dorsal penile nerve. ${ }^{30,44}$ In addition, $\alpha$-receptor-blocking agents (phenoxybenzamine) or a cystoprostatectomy cannot abolish the ejaculatory reflex with sacral motor responses. Finally, a radical prostatectomy results in the loss of 'autonomic ejaculation' but preserves 'motor ejaculation.' ${ }^{51,52}$ In conclusion, emission facilitates ejaculation but is not necessarily a trigger.

Owing to the complexity of this spinal reflex it is not always easy to predict ejaculation in neurogenic lesions. However, in patients with lesions above the thoracolumbar region the ejaculation will probably be elicited with vibratory stimulation. Patients with complete lesions at the level of or below the thoracolumbar regions are very unlikely to obtain ejaculation with vibratory stimulation. However, in $80 \%$ of all patients with a spinal cord lesion, ejaculation can be obtained with vibratory stimulation of the penis if vibrations have a frequency of at least $80 \mathrm{~Hz}$ and sufficient amplitude. ${ }^{5}$ With less intensive stimulation $(30-50 \mathrm{~Hz})$, only $50 \%$ of the patients ejaculate. This ejaculation can be facilitated with alphamimetic drugs such as eserine, midodrine or physiostigmine. $5,37,39$

Electroejaculation is electrostimulation of pelvic, hypogastric and sacral nerves and causes ejaculation in about $95 \%$ of patients with a spinal cord injury. Even in complete ablation of the sacral spinal cord but preservation of the prevertebral ganglia and the hypogastric nerve, electroejaculation can result in ejaculation. ${ }^{32}$ 
Sexual dysfunction in neurogenic patients with lesions to the spinal cord or peripheral nerves

Extrapolation of the above data to spinal cord-injured patients results in several clinical presentations. It is important to know the anatomical relations of the nervous tissue involved as well as the exact place of the motor neurons, because this largely influences the sexual dysfunctions seen.

Lesions above or at the level of the thoracolumbar region have reflex erection but no psychogenic erections. Autonomic ejaculation is possible, but nocturnal ejaculation is not. Somatic ejaculation, vibration and electroejaculation (motor neurons in ganglia and post-ganglionic fibres are intact) are possible (Figure 2a).

Lesions in between or at the level of the thoracolumbar and the sacral centres have reflex erection and should not have psychogenic erection. Nevertheless several reports are available, which indicate that these patients do have psychogenic erections. Possible explanations are neuroplasticity, PS-paravertebral ganglia and, in those below the thoracolumbar region, a decrease in OS tone. Ejaculation (autonomic and somatic and nocturnal in the case of a lesion below the thoracolumbar region) is not present and vibratory stimulation does not result in ejaculation because the reflex is interrupted; however, electroejaculation works because the postsynaptic fibres of ejaculation are intact (Figures $2 \mathrm{~b}$ and $\mathrm{c}$ ).

Lesions of the sacral centre or below can have psychogenic erections but no reflex erections. Psychogenic erections are observed and hypothetically explained by OS-erectile nerve fibres, synapses between OS and NANC, and PS-paravertebral ganglia or preserved NANC tone with decreasing OS tone. Autonomic ejaculation is not present but nocturnal ejaculation is possible. Somatic ejaculation is absent and vibratory stimulation does not result in ejaculation but electroejaculation can be successful (Figures $2 \mathrm{~d}$ and $3 \mathrm{a}$ ).
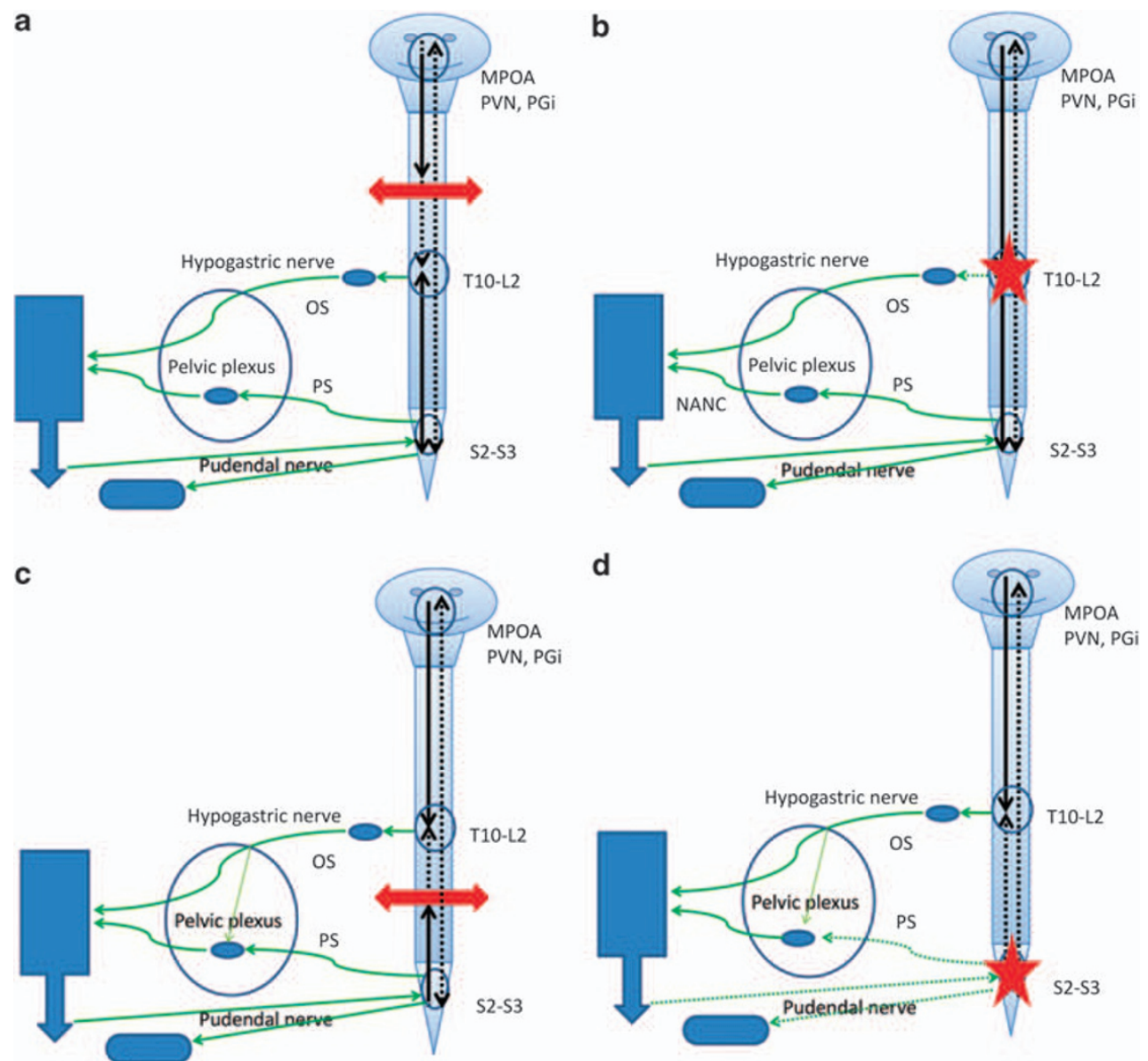

Figure 2 Neurogenic patients with lesions to the cauda equina or peripheral nerves. (a) Upper motor neuron lesion above T10. Reflex erections +; psychogenic erections -; autonomic ejaculation + (nocturnal ejaculation -); somatic ejaculation +; vibration +; electroejaculation + . (b) Spinal lesion in the thoraco-lumbar region. Reflex erections + ; psychogenic erections -; but neuroplasticity? but PS - paravertebral ganglia? Emission + (PS, neuroplasticity OS); autonomic ejaculation -; somatic ejaculation -; vibration -; electroejaculation +. (c) Spinal lesion between T10-L1 and S2-S3. Reflex erections +; psychogenic erections: +; neuroplasticity, OSerectile nerve fibres? Synapses between OS and NANC; PS - paravertebral ganglia; preserved NANC tone, erection through decreasing OS. Emission + (OS and PS); autonomic ejaculation: - but nocturnal possible; somatic ejaculation -; vibration -; electroejaculation + . (d) Lower motor neuron lesion, complete conus lesion. Reflex erections -; psychogenic erections +; neuroplasticity, OS-erectile nerve fibres? Synapses between OS and NANC; PS - paravertebral ganglia preserved NANC tone, erection through decreasing OS. emission + (OS, neuroplasticity PS). Autonomic ejaculation - but nocturnal possible; somatic ejaculation -; vibration -; electroejaculation + . 

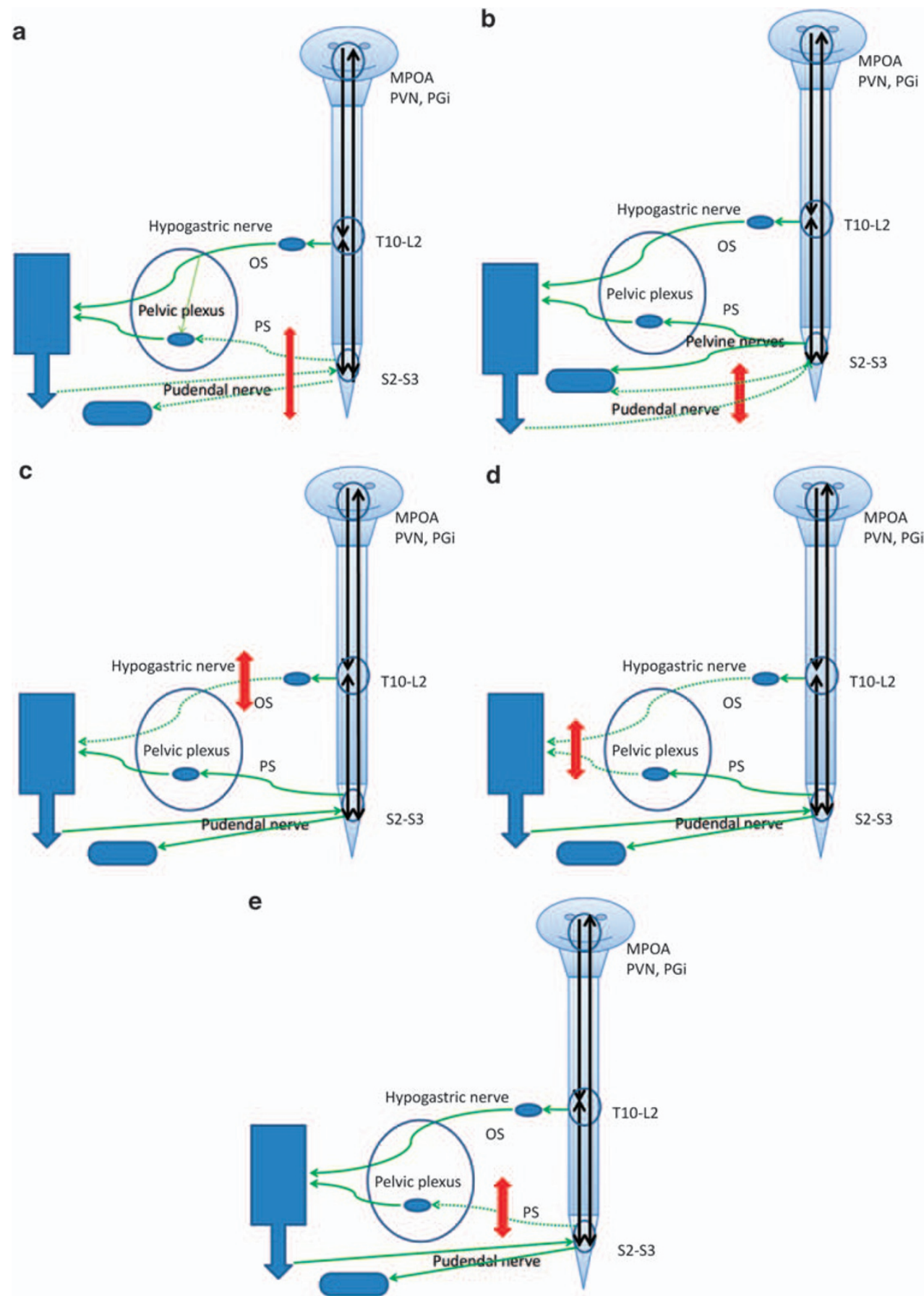

Figure 3 (a) Lower motor neuron lesion, complete cauda equina lesion. Reflex erections -; psychogenic erections: +; neuroplasticity, OSerectile nerve fibres? Synapses between OS and NANC; PS - paravertebral ganglia preserved NANC tone, erection through decreasing OS; Emission + (OS, neuroplasticity PS); autonomic ejaculation: -, nocturnal ejaculation possible; somatic ejaculation -; vibration -; electroejaculation +. (b) Bilateral pudendal nerve lesion. Psychogenic erections +; reflex erections -; emission +; autonomic ejaculation (perhaps nocturnal); somatic ejaculation - or + ; electroejaculation + ; vibration - . Some sacral innervated muscles like the levator muscles, the urethral and anal external sphincters are innervated both by the pudendal and pelvine nerves. (c) Lesions of the hypogastric nerve (anterior fixation, lymph node dissection, sigmoidectomy, congenital). Psychogenic erections + ; reflex erections + ; emission + (PS); anejaculation (autonomic); somatic ejaculation +; vibration and electroejaculation -. (d) Cystoprostatectomy. No erections; no emission; anejaculation (autonomic); somatic ejaculation +; electroejaculation -. (e) Sacrum fracture-malignancy, rectal surgery. No reflex erections; psychogenic erections? Emission + (OS, neuroplasticity PS); autonomic ejaculation + ; somatic ejaculation + ; vibration + ; electroejaculation + .

Lesions of the cauda equina result in the loss of reflex erections but the preservation of psychogenic erections (neuroplasticity, OS-erectile nerve fibres, synapses between
OS and NANC, PS-paravertebral ganglia, preserved NANC tone and erection through decreasing OS). Autonomic and somatic ejaculation disappear but nocturnal ejaculation is 
purely theoretically possible. Vibratory stimulation fails but electroejaculation works in most patients (Figure 3a). Complete lesions of both pudendal nerves (fracture of the sacrum) is a very rare condition; Alcock's syndrome is assumed to be the result of an incomplete lesion and results theoretically in the preservation of psychogenic erections, the loss of reflex erections, and autonomic (efferents intact, afferent not intact) and somatic ejaculation (but not secondary to penile stimulation, only psychogenic). Vibratory stimulation does not result in ejaculation, but electroejaculation does because the motor neurons are intact (Figures $3 a$ and $b$ ).

Lesions of the hypogastric nerve (anterior fixation, lymph node dissection, sigmoidectomy, congenital) do not result in erectile dysfunction but do result in anejaculation (autonomic) with theoretically preserved somatic ejaculation. For some, emission is an important facilitating factor and as such the somatic ejaculation can be impaired (likewise in postprostatectomy patients). Both vibratory stimulation and electroejaculation fail (Figure 3c).

Lesions of the cavernous nerves (cystoprostatectomy) lead to complete erectile dysfunction and loss of ejaculation (autonomic, also for anatomical reasons, of course). Somatic ejaculation is preserved in most patients, although in some emission is mandatory to obtain somatic ejaculation (Figure 3d).

Lesions of both sacral parasympathetic pelvic/erigent nerves (sacral fracture, malignancy, rectal surgery) result in the loss of reflex erections. There is no information on psychogenic erections in the literature, but they might be possible for already above-repeated reasons. In addition, autonomic ejaculation as somatic ejaculation must be possible (no reports found) and vibration and electroejaculation are expected to work (Figure 3e).

\section{Discussion}

As in most patients with spinal lesions or diseases, sexual function (erection (90\%), emission (100\%), ejaculation $(80 \%)$ and orgasm $(50 \%))$ is preserved to a certain extent in a majority of the cases. One might therefore expect that it makes no sense to understand the neuroanatomy of sexual function. ${ }^{1-7}$ On the contrary, however, even when sexual function is preserved to a certain extent it is altered in a significant way, and both clinicians and patients need to understand the symptoms. In addition, patients have to accept those symptoms and adapt to them. Finally, a thorough knowledge of neuroanatomy and physiology helps in understanding drug therapy and side effects. Doctors not familiar with neurological diseases might conclude that lower motor neuron lesions result in erectile dysfunction and anejaculation whereas upper motor neuron lesions do not. This review should convince them that thorough knowledge of anatomy and physiology is helpful to explain why most spinal and peripheral nerve lesions have good preservation but altered sexual function.

For many decades, a dichotomy on the neuroanatomical basis for psychogenic erection persisted. ${ }^{21}$ However, there is little evidence for the existence of a hypogastric descending pathway for psychogenic erection in a normal individual, whereas there is vast evidence for a parasympathetic origin for psychogenic erection. From a physiological point of view, it would be unexpected that a nerve-inhibiting erection (hypogastric nerve) would be capable of stimulating erection in a normal individual. Of course, neuronal plasticity can explain this but other hypotheses are possible, like synapses between OS and NANC, PS-paravertebral ganglia or preserved NANC tone. ${ }^{22,24}$

Seminal vesicles have dual (OS and PS) contractile innervations. This second dichotomy in the literature is the sympathetic and parasympathetic innervation of emission and ejaculation. This might also explain unexpected preservation of ejaculation in neurogenic patients. The dichotomy has been nicely integrated by Coolen et al. ${ }^{41}$ and can be summarized as such: emission mainly starts cholinergically (as a secretion) and mainly ends sympathetically (as a contraction), and ejaculation is mainly adrenergic and somatic.

Neuronal plasticity is an important issue in spinal cordinjured patients as they tend to survive long after their injury. ${ }^{22}$ This is quite different in diseases like amyotrophic lateral sclerosis, in which neuronal death does not always have time to develop the onset of neuronal plasticity. In spinal cord-injured patients, on the other hand, some years pass following the spinal lesion and neuronal plasticity can occur. Although unproven, this can explain several difficultto-understand clinical events such as reappearances of initial lost functions. Together with the dual innervation of the pelvic organs, neuroplasticity explains the observations that penile vibration and electroejaculation are effective in most of the patients in the long run. The minimal requirement for the success of penile vibration is a preserved reflex arch, and the minimal requirement for the success of electroejaculation is the existence of intact post-ganglionic fibres. Intact post-ganglionic parasympathetic fibres are needed for electrostimulation-evoked emission; intact post-ganglionic sympathetic fibres are the minimal requirement for successful electrostimulation-evoked ejaculation. ${ }^{22,24,25}$

Pharmacotherapy of sexual dysfunction in patients with spinal lesions is well established for erectile dysfunction. ${ }^{1,15,16}$ Some solutions are also available for premature ejaculation, such as SSRI. ${ }^{34,42,48,53,54}$ Potential new treatments for premature ejaculation to be explored are sildenafil and tramadol (SSRI-like effect). ${ }^{34,42,48,55}$ However, a valid pharmaceutical option for inducing antegrade ejaculation or treating anejaculation does not exist. Alphamimetic drugs have been tried to treat retrograde ejaculation in these patients, but no evidence of effect exists. Available drugs for facilitating ejaculation or treating anejaculation do not exist, or at least have so many side effects like physiostimine, eserine or mitodrine that they can be used for the sake of procreation, but not for improving their sexual dysfunction. ${ }^{42,48}$ More 'organ' selective drugs-and even better a combination of an adrenergic and a parasympathicomimetic drug - seem to be possible ways for improving sexual function in these neurogenic patients. In the central nervous tissue, one might try to decrease serotonin or increase 
dopamine and catecholamines for the same purpose of improving ejaculation.

\section{Conclusion}

Afferents of erection, emission and ejaculation are the pudendal nerve and descending pathways from the brain. Erection is cholinergic and NO-mediated. Emission starts cholinergically (as a secretion) and ends sympathetically (as a contraction). Ejaculation is mainly adrenergic and somatic.

In neurogenic disease, a good knowledge of neuroanatomy and physiology makes the understanding of sexual dysfunction possible and predictable. The minimal requirement for the success of penile vibration is a preserved reflex arch and the minimal requirement for the success of electroejaculation is the existence of intact post-ganglionic fibres. Intact post-ganglionic parasympathetic fibres are needed for electrostimulation-evoked emission; intact post-ganglionic sympathetic fibres are the minimal requirement for successful electrostimulation-evoked ejaculation.

\section{Conflict of interest}

The authors declare no conflict of interest.

\section{References}

1 Giuliano F, Rubio-Aurioles E, Kennelly M, Montorsi F, Kim ED, Finkbeiner AE et al. Efficacy and safety of vardenafil in men with erectile dysfunction caused by spinal cord injury. Neurology 2006; 66: 210-216.

2 Brown DJ, Hill ST, Baker HW. Male fertility and sexual function after spinal cord injury. Prog Brain Res 2006; 152: 427-439.

3 Johnson RD. Descending pathways modulating the spinal circuitry for ejaculation: effects of chronic spinal cord injury. Prog Brain Res 2006; 152: 415-426.

4 Courtois FJ, Charvier KF, Leriche A, Raymond DP. Sexual function in spinal cord injury men. I. Assessing sexual capability. Paraplegia 1993; 31: 771-784.

5 Biering-Sorensen F, Laeessoe L, Sonksen J, Bagi P, Nielsen JB, Kristensen JK. The effect of penile vibratory stimulation on male fertility potential, spasticity and neurogenic detrusor overactivity in spinal cord lesioned individuals. Acta Neurochir Suppl 2005; 93: 159-163.

6 Sonksen J, Biering-Sorensen F, Kristensen JK. Ejaculation induced by penile vibratory stimulation in men with spinal cord injuries. The importance of the vibratory amplitude. Paraplegia 1994; 32: 651-660.

7 Alexander M, Rosen RC. Spinal cord injuries and orgasm: a review. J Sex Marital Ther 2008; 34: 308-324.

8 Elliott SL. Problems of sexual function after spinal cord injury. Prog Brain Res 2006; 152: 387-399.

$9 \mathrm{Rab}$ M, Ebmer JD, Dellon AL. Anatomic variability of the ilioinguinal and genitofemoral nerve: Implications for the treatment of groin pain. Plast Reconstr Surg 2001; 108: 1618-1623.

10 Yucel S, Baskin LS. The neuroanatomy of the human scrotum: surgical ramifications. BJU Int 2003; 91: 393-397.

11 Halata Z, Munger BL. The neuroanatomical basis for the protopathic sensibility of the human glans penis. Brain Res 1986; 371: 205-230.

12 Shafik A. Pudendal canal decompression in the treatment of erectile dysfunction. Arch Androl 1994; 32: 141-149.

13 Steers WD. Neural pathways and central sites involved in penile erection: neuroanatomy and clinical implications. Neurosci Biobehav Rev 2000; 24: 507-516.
14 Giuliano F, Rampin O. Neural control of erection. Physiol Behav 2004; 83: 189-201.

15 Burnett AL. Nitric oxide regulation of penile erection: biology and therapeutic implications. J Androl 2002; 23: S20-S26.

16 Andersson KE. Pharmacology of penile erection. Pharmacol Rev 2001; 53: 417-450.

17 Courtois FJ, Macdougall JC, Sachs BD. Erectile mechanism in paraplegia. Physiol Behav 1993; 53: 721-726.

18 Giuliano F, Bernabe J, Jardin A, Rousseau JP. Antierectile role of the sympathetic nervous system in rats. J Urol 1993; 150(Part 1): 519-524.

19 Cruz MR, Liu YC, Manzo J, Pacheco P, Sachs BD. Peripheral nerves mediating penile erection in the rat. J Auton Nerv Syst 1999; 76: 15-27.

20 Sachs BD, Liu YC. Mounting and brief noncontact exposure of males to receptive females facilitate reflexive erection in rats, even after hypogastric nerve section. Physiol Behav 1998; 65: 413-421.

21 Sachs BD. Placing erection in context: the reflexogenic-psychogenic dichotomy reconsidered. Neurosci Biobehav Rev 1995; 19: 211-224.

22 McLachlan EM. Diversity of sympathetic vasoconstrictor pathways and their plasticity after spinal cord injury. Clin Auton Res 2007; 17: 6-12.

23 Andersson KE, Hedlund P, Alm P. Sympathetic pathways and adrenergic innervation of the penis. Int J Impot Res 2000; 12(S1): S5-S12.

24 Dail WG, Walton G, Olmsted MP. Penile erection in the rat: stimulation of the hypogastric nerve elicits increases in penile pressure after chronic interruption of the sacral parasympathetic outflow. J Auton Nerv Syst 1989; 28: 251-257.

25 Weihe E, Schutz B, Hartschuh W, Anlauf M, Schafer MK, Eiden LE. Coexpression of cholinergic and noradrenergic phenotypes in human and nonhuman autonomic nervous system. J Comp Neurol 2005; 492: 370-379.

26 Sipski M, Alexander CJ, Gomez-Marin O. Effects of level and degree of spinal cord injury on male orgasm. Spinal Cord 2006; 44: 798-804.

27 Shafik A. Response of the urethral and intracorporeal pressures to cavernosus muscle stimulation: role of the muscles in erection and ejaculation. Urology 1995; 46: 85-88.

28 Shafik A, El-Sibai O. Mechanism of ejection during ejaculation: identification of a urethrocavernosus reflex. Arch Androl 2000; 44: 77-83.

29 Carro-Juarez M, Rodriguez-Manzo G. The spinal pattern generator for ejaculation. Brain Res Rev 2008; 58: 106-120.

30 Carro-Juarez M, Rodriguez-Manzo G. Sensory and motor aspects of the coital reflex in the spinal male rat. Behav Brain Res 2000; 108: $97-103$.

31 Brindley GS. The fertility of men with spinal injuries. Paraplegia 1984; 22: 337-348.

32 Seager SW, Halstead LS. Fertility options and success after spinal cord injury. Urol Clin North Am 1993; 20: 543-548.

33 Vanden Broucke H, Everaert K, Peersman W, Claes H, Vanderschueren D, Van Kampen M. Ejaculation latency times and their relationship to penile sensitivity in men with normal sexual function. J Urol 2007; 177: 237-240.

34 Kuhr CS, Heiman J, Cardenas D, Bradley W, Berger RE. Premature emission after spinal cord injury. J Urol 1995; 153: 429-431.

35 Bell C, McLean JR. Localization of norepinephrine and acetylcholinesterase in separate neurons supplying the guinea-pig vas deferens. J Pharmacol Exp Ther 1967; 157: 69-73.

36 Wieder JA, Brackett NL, Lynne CM, Green JT, Aballa TC. Anesthetic block of the dorsal penile nerve inhibits vibratoryinduced ejaculation in men with spinal cord injuries. Urology 2000; 55: 915-917.

37 Brackett NL, Ferrell SM, Aballa TC, Amador MJ, Padron OF, Sonksen J et al. An analysis of 653 trials of penile vibratory stimulation in men with spinal cord injury. J Urol 1998; 159: 1931-1934.

38 Brindley GS. Reflex ejaculation under vibratory stimulation in paraplegic men. Paraplegia 1981; 19: 299-302.

39 Bird VG, Brackett NL, Lynne CM, Aballa TC, Ferrell SM. Reflexes and somatic responses as predictors of ejaculation by penile 
vibratory stimulation in men with spinal cord injury. Spinal Cord 2001; 39: 514-519.

40 Giuliano F, Rampin O. Central neural regulation of penile erection. Neurosci Biobehav Rev 2000; 24: 517-533.

41 Coolen LM, Allard J, Truitt WA, McKenna KE. Central regulation of ejaculation. Physiol Behav 2004; 83: 203-215.

42 Giuliano F, Clement P. Physiology of ejaculation: emphasis on serotonergic control. Eur Urol 2005; 48: 408-417.

43 Kolbeck SC, Steers WD. Neural regulation of the vas deferens in the rat: an electrophysiological analysis. Am J Physiol 1992; 263 (Part 2): R331-R338.

44 Kimura Y. On peripheral nerves controlling ejaculation. Tohoku J Exp Med 1971; 105: 177-190.

45 Kihara K, de Groat WC. Sympathetic efferent pathways projecting bilaterally to the vas deferens in the rat. Anat Rec 1997; 248: 291-299.

46 Kihara K, de Groat WC. Sympathetic efferent pathways projecting to the bladder neck and proximal urethra in the rat. J Auton Nerv Syst 1997; 62: 134-142.

47 Fedan JS, Besse JC, Carpenter FG, Teague RS. Motor innervation of the smooth muscle of the rat seminal vesicle. J Pharmacol Exp Ther 1977; 201: 285-297.

48 Hib J, Ponzio R, Vilar O. Effects of autonomic drugs on contractions of rat seminal vesicles in vivo. J Reprod Fertil 1984; 70: $197-202$.
49 Hsieh JT, Chien CT, Liu SP, Chen CF, Lai MK. An experimental model to evaluate the in vivo response of rat seminal vesicle to electrical stimulation. Neurosci Lett 1996; 204: 215-217.

50 Kamischke A, Nieschlag E. Treatment of retrograde ejaculation and anejaculation. Hum Reprod Update 1999; 5: 448-474.

51 Bergman B, Nilsson S, Petersen I. The effect on erection and orgasm of cystectomy, prostatectomy and vesiculectomy for cancer of the bladder: a clinical and electromyographic study. Br J Urol 1979; 51: 114-120.

52 Barnas JL, Pierpaoli S, Ladd P, Valenzuela R, Aviv N, Parker M et al. The prevalence and nature of orgasmic dysfunction after radical prostatectomy. BJU Int 2004; 94: 603-605.

53 Hsieh JT, Chang HC, Law HS, Hsieh $\mathrm{CH}$, Cheng JT. In vivo evaluation of serotonergic agents and alpha-adrenergic blockers on premature ejaculation by inhibiting the seminal vesicle pressure response to electrical nerve stimulation. Br J Urol 1998; 82: 237-240.

54 Hsieh JT, Liu SP, Hsieh CH, Cheng JT. An in vivo evaluation of the therapeutic potential of sympatholytic agents on premature ejaculation. BJU Int 1999; 84: 503-506.

55 Chen J, Mabjeesh NJ, Matzkin H, Greenstein A. Efficacy of sildenafil as adjuvant therapy to selective serotonin reuptake inhibitor in alleviating premature ejaculation. Urology 2003; 61: 197-200. 